\title{
The Relation Between Field Massive Stars and Clusters
}

\author{
M. S. Oey ${ }^{1}$, N. L. King ${ }^{2}$, J. Wm. Parker ${ }^{3}$ and J. B. Lamb ${ }^{1}$ \\ ${ }^{1}$ University of Michigan, Department of Astronomy, Ann Arbor, MI 48109-1042, USA \\ ${ }^{2}$ Lowell Observatory, 1400 W. Mars Hill Rd., Flagstaff, AZ 86001, USA \\ ${ }^{1}$ Southwest Research Institute, Suite 426, 1050 Walnut St., Boulder, CO 80302, USA
}

\begin{abstract}
Massive "field" stars are those that appear in apparent isolation, in contrast to those in clusters. Whereas cluster stars are formed together in large aggregates, simultaneously, field stars have multiple origins. Some massive field stars may be the "tip of the iceberg" on small groups of physically associated stars, while others appear to be "runaway" stars that are dynamically ejected from clusters. What is the intrinsic relation between clusters and field stars, and what is the faction of runaway stars? Since massive stars are the most luminous stellar population, their demographics are accessible in the nearest external galaxies. We present our current efforts to understand these issues for the Small Magellanic Cloud.
\end{abstract}

Keywords. open clusters and associations: general, stars: early-type, stars: kinematics

\section{Introduction}

Runaway $\mathrm{OB}$ stars are an important probe of the binary fractions and dynamical properties of their parent clusters. Dynamical ejection of massive stars, dominated by binary-binary interactions (e.g., Mikkola 1983; Leonard \& Duncan 1988), are diagnostic of cluster core densities and binary parameters like binary fraction, hardness, and mass ratios. Supernova "slingshot" ejection also contributes to the runaway population, and is also strongly dependent on properties of the parent binary population (e.g., Stone 1982; Portegies Zwart 2000). A number of studies have compared predicted massive star ejection rates and properties with those of observed runaways (e.g., Gies \& Bolton 1986; Hoogerwerf et al. 2000). However, there are relatively few observations of runaway OB stars as populations, and therefore their statistical properties are uncertain.

To study the properties of any runaway population, it is essential to understand the entire OB field and to accurately distinguish contributions from different origins. In addition to runaways, an empirically-defined sample of field OB stars also includes stars in small groups that have no other observed massive star. There may also be high-mass stars that formed in true isolation, although if they exist, they may be extremely rare.

To date, all studies of runaway OB stars consider samples in the broader solar neighborhood (e.g., Gies \& Bolton 1986; Hoogerwerf et al. 2000; de Wit et al. 2005). As a complementary effort, we are carrying out a comprehensive study of the field massive stars in the nearby Small Magellanic Cloud (SMC). Our sample of photometrically identified field OB candidates is defined by applying a friends-of-friends algorithm (Battinelli 1991) to the $U B V R$ survey of the SMC by Massey (2002). A second sample of O-star candidates is identified by combining these data with the $1625 \AA$ imaging of the SMC Bar by the Ultraviolet Imaging Telescope (Parker et al. 1998). Candidate stars having no other candidate within clustering lengths of $28 \mathrm{pc}$ and $34 \mathrm{pc}$ for the OB and O-star samples, respectively, are defined to be field massive stars. These represent essentially complete samples of field $O B$ star candidates across the spatial extent of these surveys. 

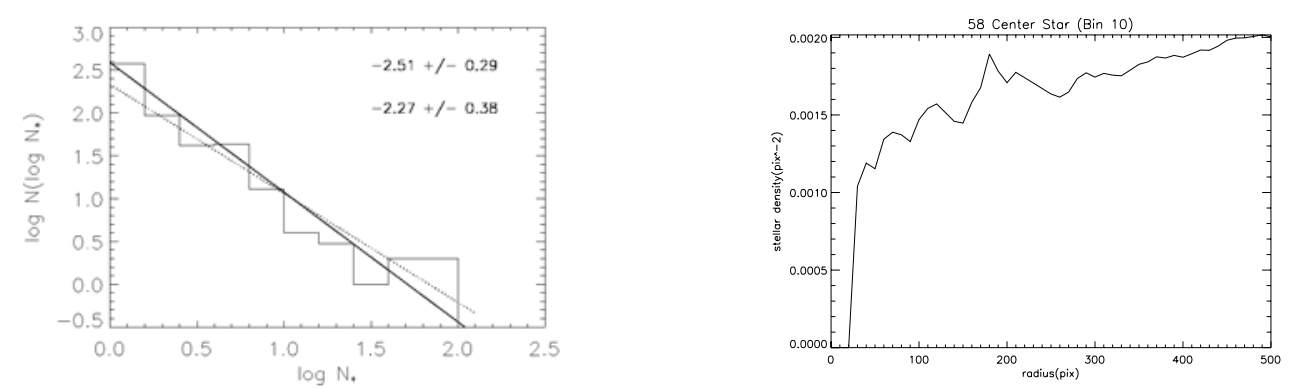

Figure 1. Left panel: Clustering law for OB sample (from Oey et al.2004). Right panel: Stellar density vs radius near the O star AZV 58 from HST/ACS F814W imaging.

This analysis also yields the SMC clusters and the distribution in the number of candidates $N_{*}$ per cluster, for both samples. Both are broadly consistent with the universal $N\left(N_{*}\right) \propto N_{*}^{-2}$ clustering law (e.g., Hunter et al. 2003). The individual field stars, corresponding to $N_{*}=1$, fall smoothly onto this distribution, strongly suggesting that the majority of these stars are the "tip of the iceberg" on small stellar groups of lower-mass stars, having no other candidate massive stars (Fig. 1 left panel; see Oey, King \& Parker 2004 for details).

Nevertheless, a significant fraction $(\lesssim 50 \%)$ of our field massive stars could be runaways and still be statistically consistent with the smooth appearance of the clustering law. Our HST/ACS SNAP program yielded imaging of 7 field O stars in F555W and F814W, to confirm whether the majority indeed have lower-mass companions. Our preliminary results of the stellar density profiles show that about half of the fields show the existence of small groups, while half appear to be truly isolated stars (Fig. 1 right panel).

We are also carrying out a complete spectroscopic survey of the SMC Bar with the Magellan IMACS multi-object spectrograph. These will yield radial velocity measurements to constrain the fraction of runaways (see Lamb \& Oey, this volume), as well as confirm exact spectroscopic types. Although full space velocities are not available, the statistics of the radial velocities will yield strong constraints on the properties of both the runaway and in situ field stars.

\section{Acknowledgements}

We acknowledge NASA ADP grant NAG5-9248 and NASA HST-GO-10629.01.

\section{References}

Battinelli, P. 1991, A\&A 244, 69

de Wit, W. J., Testi, L., Palla, F., \& Zinnecker, H. 2005, A\&A 437, 247

Gies, D. R. \& Bolton, C. T. 1986, ApJS 61, 419

Hoogerwerf, R., de Bruijne, J. H. J., \& de Zeeuw, P. T. 2001, A $\& A$ 365, 49

Hunter, D. A., Elmegreen, B. G., Dupuy, T. J., \& Mortonson, M. 2003, AJ 126, 1836

Leonard, P. J. T. \& Duncan, M. J. 1988, AJ 96, 222

Massey, P. 2002, ApJS, 141, 81

Mikkola, S. 1983, MNRAS 203, 1107

Oey, M. S., King, N. L., \& Parker, J. W. 2004, $A J$ 127, 1632

Parker, J. W., et al. 1998, AJ 116, 180

Portegies Zwart, S. F. 2000, ApJ 544, 437

Stone, R. C. $1982, A J$ 87, 90 